\section{P450 PREP USE, STD ACQUISITION AND SEXUAL RISK BEHAVIOR}

${ }^{1}$ Amy Burrell ${ }^{*},{ }^{2}$ Carla Tilchin, ${ }^{3}$ Sebastian Ruhs, ${ }^{4}$ Christina Schumacher, ${ }^{5}$ Errol Fields, ${ }^{6}$ Jessica Wagner, ${ }^{7}$ Adena Greenbaum, ${ }^{2}$ Jacky Jennings. ${ }^{1}$ Chase Brexton Health Services, Baltimore, USA; ${ }^{2}$ Johns Hopkins University School of Medicine, Center for Child and Community Health Research (CCHR), Baltimore, USA; ${ }^{3}$ Chase Brexton, Baltimore, USA; ${ }^{4}$ Johns Hopkins School of Medicine, Center for Child and Community Health Research, Baltimore, USA; ${ }^{5} J o h n s$ Hopkins School of Medicine, Baltimore, USA; ${ }^{6} J o h n s$ Hopkins University, Baltimore, USA; ${ }^{7}$ Baltimore City Health Department, Baltimore, USA

10.1136/sextrans-2019-sti.534

Background Some studies have shown an association between HIV pre-exposure prophylaxis (PrEP) use, STD acquisition and increased sexual risk behaviors (e.g. condomless sex). The objective was to determine the association between PrEP use, STD infection (i.e. syphilis, gonorrhea, chlamydia) and sexual risk behaviors (i.e. condomless sex, sex partner concurrency) in one mid-Atlantic city with an established epidemic of HIV.

Methods Data came from the Understanding Sexual Health in Networks Study (USHINE), an ongoing longitudinal cohort study of MSM between the ages of 18-45. Participants completed an egocentric sexual network survey. Summary statistics, chi-squared tests, and t-tests were used for hypothesis testing. Results 173 men completed the sexual network survey. 52 (30.1\%) men were HIV uninfected and 52 (30.1\%) men reported PrEP use. PrEP users (vs non-users) were less likely to be positive for syphilis ( $10.6 \%$ vs $26.8 \%, \mathrm{p}=0.03)$, but no more likely to be positive for gonorrhea $(15.4 \%$ vs $8.0 \%$, $\mathrm{p}=0.25)$ or chlamydia $(17.3 \%$ vs $16.0 \%, \mathrm{p}=0.86)$. PrEP users (vs non-users) were more likely to report condomless sex $(88.5 \%$ vs $69.2 \%, p=0.02)$ and sex partner concurrency $(57.7 \%$ vs $32.7 \%, p=0.01)$.

Conclusion PrEP users were less likely to be infected with syphilis and no more likely than non-PrEP users to be gonorrhea and chlamydia infected. PrEP users were more likely to report sexual risk behaviors including condomless sex and sex partner concurrency compared to non-PrEP users. The increased sexual risk behaviors among PrEP users is concerning and emphasizes the importance of prevention messaging for PrEP users.

Disclosure No significant relationships.

\section{P452 A NURSE-LED HIV PRE-EXPOSURE PROPHYLAXIS PROGRAM AT COOL AID COMMUNITY HEALTH CENTRE FOR MEN WHO HAVE SEX WITH MEN}

Karen Lundgren*. Cool Aid Community Health Centre, Victoria, Canada

\subsection{6/sextrans-2019-sti.535}

Background Gay, bisexual and MSM continue to comprise the greatest number of new HIV diagnosis in BC (BCCDC, 2016). The complexity of the HIV epidemic among MSM has highlighted the need for broader approaches to HIV prevention. Publicly funded access to PrEP in BC began in 2018 . The low barrier MSM STI testing clinic at Cool Aid CHC demonstrates that PrEP can be implemented successfully through a nurse-led program.

Methods Description of model of care/intervention: This novel CHC based Men's STI Testing Clinic is staffed by STI certified practice nurses and run in partnership with AIDS
Vancouver Island (AVI). It is advertised through MSM social media sites by the AVI Men's Wellness Coordinator. At the initial visit, clients complete a HIRI-MSM index, a sexual/ medical history, required screening and participate in PrEP counseling and education. Physicians review the results and sign the PrEP prescription. Clients without primary care are accepted as patients at the clinic.

Results Effectiveness: Our innovative non-judgemental, nurseled model has removed barriers to sexual health screening and enrolled 124 MSM in the PrEP program. Initial PrEP screening showed an STI incidence of $19 \%$ and a previous syphilis diagnosis in $15 \%$ of those screened. After PrEP initiation STI incidence increased by $7 \%$, highlighting the need for continued safer sex education. There have been no HIV infections amongst PrEP recipients. 20\% have discontinued PrEP after approval. 56\% of those enrolled felt unable to access PrEP through their physician. 44\% of PrEP clients are now linked to primary care at $\mathrm{Cool}$ Aid $\mathrm{CHC}$, demonstrating that PrEP can be a tool to prevent HIV transmission in MSM, while engaging patients in primary care and regular STI screening. Conclusion and next steps: This innovative program increased access to PrEP for MSM, increased STI testing/treatment, helped to prevent HIV transmission and linked clients to primary care.

Disclosure No significant relationships.

\section{P453 INCIDENCE AND PREDICTORS OF URETHRAL AND RECTAL CHLAMYDIA AND GONORRHEA AMONG MEN WHO HAVE SEX WITH MEN TAKING PREP IN KENYA}

${ }^{1}$ Supriya Mehta*, ${ }^{2}$ Duncan Okal, ${ }^{3}$ Susan Graham, ${ }^{2}$ George N'Gety, ${ }^{2}$ Eve Obondi, ${ }^{4}$ Robert Bailey, ${ }^{2}$ Fredrick Otieno. 'University of Illinois at Chicago, Epidemiology and Biostatistics, Chicago, USA; ${ }^{2}$ Nyanza Reproductive Health Society, Kisumu, Kenya; ${ }^{3}$ University of Washington, Seattle, USA; ${ }^{4}$ University of Illinois at Chicago, Chicago, USA

\subsection{6/sextrans-2019-sti.536}

Background We measured the incidence and associated factors of urethral and rectal C. trachomatis (CT) and N. gonorrhoeae (NG) among a Kenyan cohort of MSM taking PrEP.

Methods Enrolled MSM underwent audio computer assisted self-interview for behaviour and socio-demographics, with medical examination every 6 months. CT and NG were diagnosed by polymerase chain reaction assay in urine and rectal swabs at baseline, 6- and 12- months. We identified factors associated with incident urethral infection using multivariable Cox regression and report adjusted hazard ratios (aHR).

Results October 2017 - January 2018, 158 participants were enrolled and initiated PrEP. Follow-up was 99\% (month 6) and $93 \%$ (month 12). At baseline, 10.4\% had urethral CT/ NG (either or both infections), and 3.3\% had rectal CT/NG, with total $11.4 \%$ prevalence CT/NG. At 6 months, CT/NG was $7.7 \%$ (urethral) and $0 \%$ (rectal), and $10.2 \%$ (urethral) and $0 \%$ (rectal) at 12 months. There were 22 incident infections (19 CT, 2 NG, 1 CT and NG) during 152 person-years (py) follow-up, with no re-infections (13.9 cases/100 py; 95\% CI: 9.2-21.1). Risk decreased $12 \%$ per one year increase in age $(\mathrm{aHR}=0.88, \mathrm{p}=0.012)$ and was $73 \%$ lower for men reporting last sex partner being female $(\mathrm{aHR}=0.37, \mathrm{p}=0.019)$. For each one unit increase in social support, men had $3 \%$ 